\title{
Weakly nonlinear ion sound waves in gravitational systems
}

\author{
P. Guio $\odot^{1,2}$ and H. L. Pécseli $\odot^{2,3}$ \\ ${ }^{1}$ Department of Physics and Astronomy, University College London, London WC1E 6BT, United Kingdom \\ ${ }^{2}$ Arctic University of Norway, Department of Physics and Technology, N-9037 Troms $\phi$, Norway \\ ${ }^{3}$ University of Oslo, Physics Department, Box 1048 Blindern, N-0316 Oslo, Norway
}

(Received 20 December 2019; accepted 24 March 2020; published 27 April 2020)

\begin{abstract}
Ion sound waves are studied in a plasma subject to gravitational field giving rise to vertically inhomogeneous steady-state plasma conditions. Such systems are interesting by exhibiting a wave growth that is a result of energy flux conservation for pulses propagating in an inhomogeneous system. The increase of the amplitude of a pulse as it propagates along the density gradient in the direction of decreasing density gives rise to an enhanced interaction between waves and plasma particles that can be modeled by a modified Korteweg-de Vries equation. Analytical results are compared with numerical particle-in-cell simulations of the problem. Our code assumes isothermally Boltzmann distributed electrons resulting in a nonlinear Poisson equation. The ion component is treated as a collection of individual particles interacting through collective electric fields. Deviations from quasineutrality are allowed for.
\end{abstract}

DOI: 10.1103/PhysRevE.101.043210

\section{STEADY STATE}

We consider a hot plasma in a gravitational field in the vertical $z$ direction, with the gravitational acceleration $\mathbf{g}$ pointing in the downwards direction. Steady-state solutions with $\bar{u}_{z}=0$ are readily obtained for the case where we have a balance between the gravitational effect and particles' thermal pressure. We introduce $\bar{\phi}(z)$ for the spatially varying timeindependent electrostatic potential, $-e$ for the electron charge, and $M$ for a reference ion mass. In terms of these we have for our case $\bar{\phi}=-z M g / e$ and the steady-state vertical electric field is constant, $\overline{\mathbf{E}}=\widehat{\mathbf{z}} M g / e$ in the positive $z$ direction so that the constant gravitational force is balanced by the ambipolar electric field induced by the charge separation caused by the finite electron pressure. This is incidentally an interesting result: With a constant electric field we have here the right-hand side of Poisson's equation $\nabla \cdot \mathbf{E}=e\left(n_{i}-n_{e}\right) / \varepsilon_{0}$ to vanish identically, so that the steady-state solution is quasineutral, $n_{e}=n_{i}$, even though no assumption of quasineutrality was made explicitly. For the plasma density we find

$$
\bar{n}(z)=n_{0} \exp \left(-z g / C_{s}^{2}\right),
$$

with $C_{s} \equiv \sqrt{T_{e} / M}$ being the ion sound speed, here for cold ions and warm electrons. We can introduce a vertical scale length $L_{g} \equiv C_{s}^{2} / g$. Temperatures are in energy units, i.e., without Boltzmann's constant.

More generally both ions and electrons will contribute. The classical and simplest of these equilibrium solutions $[1,2]$ are found for isothermal conditions $T_{e}=T_{i} \equiv T$, with the plasma density varying as $\bar{n}=n_{0} \exp \left(-\frac{1}{2} z(m+M) g / T\right)$. In this case the constant gravitational acceleration we have $\bar{n}(m+M) g$ to balance the plasma pressure $2 T d \bar{n} / d z$. The effect of gravity on the electrons is negligible, but they respond to the collective electric fields. For steady-state solutions we can assume both electrons and ions to be in an isothermal Boltzmann equilibrium, possibly with different temperatures, i.e.,

$$
n_{e}=n_{0 e} \exp \left(e \phi / T_{e}\right),
$$

and

$$
n_{i}=n_{0 i} \exp \left(\frac{-e \phi-M g z}{T_{i}}\right) .
$$

We can impose neutrality at the position where $\phi=0$, taken to be $z=0$, to give $n_{0 e}=n_{0 i} \equiv n_{0}$. To determine the electrostatic potential we can then insert into Poisson's equation $\nabla^{2} \phi=e\left(n_{e}-n_{i}\right) / \varepsilon_{0}$ to give $e \phi=-M g z T_{e} /\left(T_{i}+T_{e}\right), E=$ $g(M / e) T_{e} /\left(T_{i}+T_{e}\right)=$ const. and $n_{e}=n_{0} \exp \left(-z M g /\left(T_{i}+\right.\right.$ $\left.\left.T_{e}\right)\right), n_{i}=n_{0} \exp \left(-z M g /\left(T_{e}+T_{i}\right)\right)$, i.e., $n_{i}=n_{e}$ also for $T_{i} \neq$ 0 . The present results contain the Rosseland-Pannekoek isothermal equilibrium [1,2] as a special limit. In principle, the results are correct for any intensity of the gravitational field.

The steady-state solution outlined here assumes one ion species only. If we insert another singly charged lighter ion species the gravitational force is smaller on this, while the force from the vertical electric field is the same. This lighter species will consequently be accelerated in the vertical direction to give the "polar wind" [3]. Conceptually, the spatial separation between the free electrons and the heavier gravitationally bound ions results in an ambipolar electric field, which continuously accelerates light ions upward in the polar ionosphere [4]. In the present study we will discuss another form of acceleration found in collisionless gravitational plasmas and restrict the analysis to one ion species. A magnetic field can be present and is then assumed to be vertical, as, for instance, near the magnetic poles of Earth and planets with dipolar magnetic field such as Jupiter or Saturn.

The present study assumes collisionless plasmas. Inclusion of collisions with a neutral component [5] in the model is possible, but is not considered here. Inhomogenous temperature along magnetic field lines is relevant in naturally occurring 


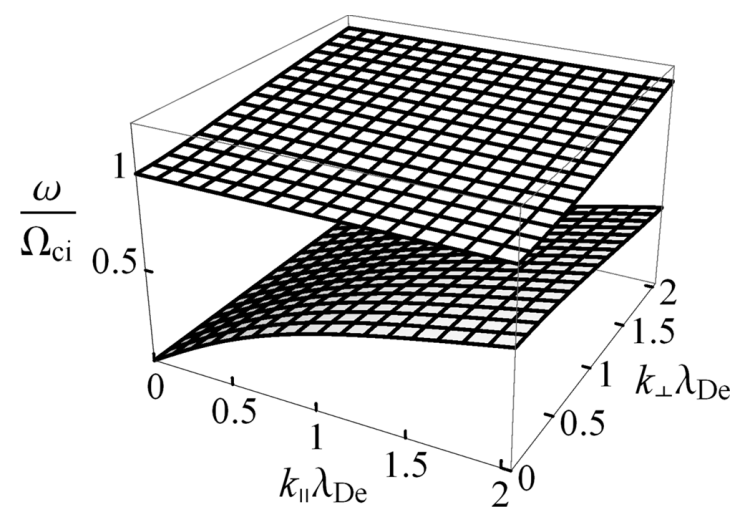

FIG. 1. Linear dispersion relation for ion waves propagating in a homogeneous magnetized plasma with $\Omega_{c i}>\Omega_{p i}$. There are two branches: a low frequency branch $\omega<\Omega_{p i}$ relevant here, and a high frequency wave component $\omega \approx \Omega_{c i}$. We have $\Omega_{c i}=2 \Omega_{p i}$, and $T_{e}=10 T_{i}$.

plasmas, and in cases where, for instance, the ion temperature decreases for increasing altitude we can expect unstable conditions somewhat equivalent to interchange modes [6]. In a neutral atmosphere the equivalent would be a complex Brunt-Väisälä frequency [7]. Temperature variations along magnetic field lines will require sources and sinks due to the high thermal conductivity of collisionless plasmas. These conditions are not included in the present study, where we address the basic concepts of charged particle acceleration by weakly nonlinear electrostatic plasma pulses. The analysis in this work refers to plasmas in gravitational fields in part because it is mathematically the most attractive. The basic results and ideas being developed will, however, have a wider range of applications [8-10].

\section{LINEAR WAVE PROPAGATION}

This section summarizes the properties of linear wave propagation. A strictly one-dimensional model has limited relevance for realistic applications. The presence of a magnetic field need not be sufficient to justify such a model, in particular also because application of the results will generally imply that the perturbations also have a component perpendicular to the magnetic field. As a reference case we include a summary for low frequency waves propagating in a homogeneous magnetized plasma.

\section{A. Homogeneous magnetized plasma conditions}

For homogeneous magnetized plasma conditions the linear dispersion relation $\omega=\omega(\mathbf{k})$ can be found in the literature [11]. Two limiting cases can be recognized: $\Omega_{c i}>\Omega_{p i}$ and $\Omega_{c i}<\Omega_{p i}$ in terms of ion cyclotron and ion plasma frequencies. There is a significant difference between these two cases, which is best appreciated by comparing their linear dispersion relations [11]. A previous study [12,13] discussed weakly nonlinear ion waves for the case where $\Omega_{c i}<\Omega_{p i}$. The other limit with $\Omega_{c i}>\Omega_{p i}$ will be relevant for the present analysis. The linear dispersion relation and the variation of the group velocity vectors for this latter case is shown in Figs. 1 and 2.

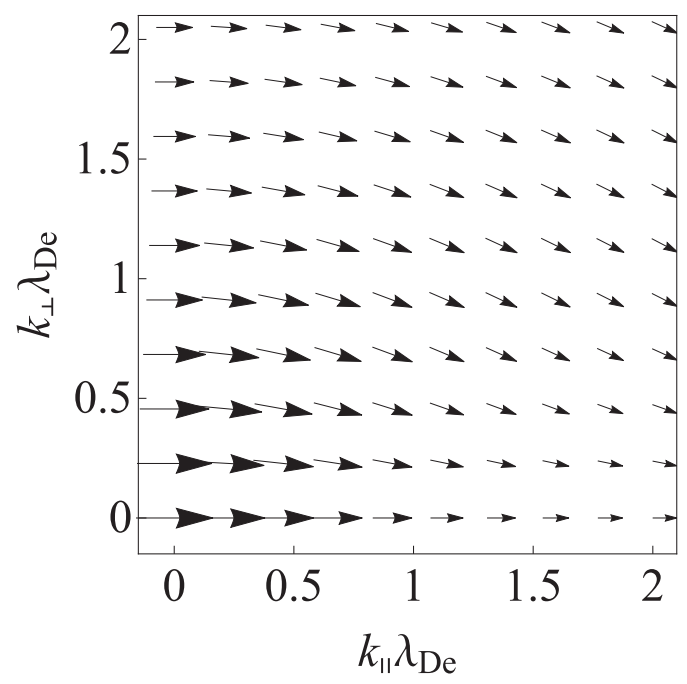

FIG. 2. The variation (direction and magnitude) of the group velocity vectors for the low frequency branch from Fig. 1.

We find that the group velocity vectors are nearly parallel to $\mathbf{B}$ for the low frequency branch. A localized perturbation will therefore propagate along magnetic field lines with small dispersion in the direction $\perp \mathbf{B}$ for this wave type. The wave forms analyzed in the following belong to the low frequency branch. A spatially one-dimensional study is justified by considering conditions where a waveguide mode excited in a magnetic flux tube with enhanced electron temperatures $T_{e} \gg$ $T_{i}$ compared to the surrounding plasma similar to a previous study [13].

\section{B. Inhomogenous plasma conditions with gravity}

Propagation of waves in a gravitational field in a horizontally striated environment has an equivalent in the neutral atmosphere $[14,15]$ where a vertical density gradient is found, and the problem has similarities with the one considered in the present study. Here we use the linearized ion continuity equation and momentum equations first for cold ions for illustration. Introduce the potential as $\phi=\bar{\phi}+\widetilde{\phi}$ and $n=$ $\bar{n}(z)+\widetilde{n}$ to separate the fluctuating parts from the steady-state equilibrium values. With the present assumptions, the velocity $u_{z}$ has fluctuating components only so $\sim$ is omitted here. Assume also Boltzmann distributed electrons, $n_{e}=\bar{n}_{e}(z)+\widetilde{n}_{e}=$ $n_{0} \exp \left(e \phi / T_{e}\right)=n_{0} \exp \left(e(\bar{\phi}+\widetilde{\phi}) / T_{e}\right)$, and quasineutrality, $n_{e} \approx n_{i} \equiv n$. The reference density $n_{0}$ is found where the potential $\bar{\phi}$ vanishes at steady state and corresponds to $\bar{n}_{e}(z=0)$. Linearizing the electron equation we have $\tilde{n}_{e}=$ $n_{0}\left(e \widetilde{\phi} / T_{e}\right) \exp \left(e \bar{\phi} / T_{e}\right) \equiv \bar{n}(z) e \widetilde{\phi} / T_{e}$ giving the linear ion continuity equation in the form,

$$
\frac{e}{T_{e}} \frac{\partial \tilde{\phi}}{\partial t}=-\frac{\partial u_{z}}{\partial z}+\frac{u_{z}}{L_{g}}
$$

and the linear ion momentum equation for cold ions becomes

$$
\frac{\partial u_{z}}{\partial t}=-\frac{e}{M} \frac{\partial \tilde{\phi}}{\partial z} .
$$


Eliminating $\widetilde{\phi}$ we find for a plane wave solution $\exp \left(-i\left(\omega t-k_{z} z\right)\right)$ a complex dispersion relation in the form,

$$
\omega^{2}-i g k_{z}-C_{s}^{2} k_{z}^{2}=0
$$

where $g$ is the gravitational acceleration, here taken constant. If we assume an initial perturbation with real $k$ we find a complex frequency,

$$
\omega= \pm \sqrt{i g k_{z}+C_{s}^{2} k_{z}^{2}} .
$$

The interesting feature is that plane waves propagating in the positive $z$ direction appear to be unstable, while waves propagating in the opposite direction are damped. As stated, this refers to a plane wave excited initially. It is here even more interesting to have a wave excited at a boundary say at $z=0$ with a real frequency $\omega$, and investigate its spatial variation. For this problem we have from (4) the result,

$$
k_{z}=-\frac{1}{2 C_{s}^{2}}\left(i g \pm \sqrt{4 C_{s}^{2} \omega^{2}-g^{2}}\right) .
$$

The spatial variation of, for instance, the fluctuating linear ion fluid velocity will be given by $\exp \left(-i\left(\omega t-k_{z} z\right)\right)$, or

$$
\begin{aligned}
u_{z}(z, t)= & U_{0} \exp \left(\frac{1}{2} z g / C_{s}^{2}\right) \\
& \times \exp \left( \pm i \frac{z}{2 C_{s}^{2}} \sqrt{4 C_{s}^{2} \omega^{2}-g^{2}}\right) \exp (-i \omega t),
\end{aligned}
$$

showing that the wave increases in amplitude as it propagates upwards in the vertical direction for $z>0$. For downwards direction of propagation, $z<0$ we find a wave damping. Note the cutoff at $\omega_{c}=\frac{1}{2} g / C_{s}$. For real $\omega$ and complex $k$ we have no wave propagation for $\omega<\omega_{c}$.

The system is not unstable in a strict sense since there is no free energy to drive an instability. A physical argument for the observed wave growth can be given by considering the lowest order contribution to the kinetic wave energy density $\frac{1}{2} \bar{n} M u_{z}^{2}$. The wave energy density flux is then to the same accuracy $\frac{1}{2} \bar{n} M u_{z}^{2} C_{s}$ with a constant $C_{s}$ for the given conditions. Since $\bar{n} \rightarrow 0$ for $z \rightarrow \infty$ we must at the same time have $u_{z}^{2} \rightarrow \infty$ to keep the flux constant. The time averaged wave energy density flux is for $\omega \gg \omega_{c}$ given as $\frac{1}{2} \bar{n} M\left|u_{z}\right|^{2} C_{s} \approx$ $\frac{1}{2} n_{0} M C_{s} U_{0}^{2}=$ constant since the $z$ variation from $\bar{n}$ cancels the $z$ variation from $\left|u_{z}\right|^{2}$, as expected. The analysis of the potential energy associated with the wave can be analyzed in the same manner. The argument cannot readily be applied to the initial value problem: If we in that case take a plane wave at $t=0$, the initial wave energy density will be inhomogeneously distributed.

\section{CONSEQUENCES OF FINITE ION TEMPERATURES AND DEVIATIONS FROM QUASINEUTRALITY}

\section{A. Finite ion temperatures}

A finite ion temperature changes the isothermal steadystate solution to $T_{i} \ln \bar{n}(z)=-e \bar{\phi}(z)-M g z$ for the ions so that

$$
\bar{n}(z)=n_{0} \exp \left(-z \frac{M g}{T_{e}+T_{i}}\right)
$$

and $e \bar{\phi}(z)=-z M g T_{e} /\left(T_{e}+T_{i}\right)$, giving a modified expression for the steady-state vertical length scale $L_{g}=\left(T_{e}+T_{i}\right) /(M g)$ accounting for a finite ion temperature $T_{i}$.

The basic equations are as follows. Introducing $\eta \equiv \tilde{n}_{i} / \bar{n}$, the linearized ion continuity equation is

$$
\frac{\partial \eta}{\partial t}+u_{z} \frac{d \ln \bar{n}}{d z}+\frac{\partial u_{z}}{\partial z}=0,
$$

where $d \ln \bar{n} / d z=-1 / L_{g}$.

With $p_{i}=\bar{p}_{i}(z)+\widetilde{p}_{i}, \phi=\bar{\phi}(z)+\widetilde{\phi}, n=\bar{n}(z)+\widetilde{n}$, etc., we can write the ion momentum equation as

$$
\begin{aligned}
M \frac{D u_{z}}{D t}= & -\frac{1}{\bar{n}(z)+\widetilde{n}} \frac{\partial\left(\bar{p}_{i}(z)+\widetilde{p}_{i}\right)}{\partial z} \\
& -e \frac{\partial(\bar{\phi}(z)+\widetilde{\phi})}{\partial z}-M g .
\end{aligned}
$$

Ignoring products of small terms we find

$$
\begin{aligned}
M \frac{\partial u_{z}}{\partial t}= & -\frac{1}{\bar{n}(z)}\left(1-\frac{\tilde{n}}{\bar{n}(z)}\right) \frac{\partial\left(\bar{p}_{i}(z)+\tilde{p}_{i}\right)}{\partial z} \\
& -e \frac{\partial(\bar{\phi}(z)+\widetilde{\phi})}{\partial z}-M g \\
= & -\frac{1}{\bar{n}(z)} \frac{\partial \bar{p}_{i}(z)}{\partial z}+\frac{\tilde{n}}{\bar{n}^{2}(z)} \frac{\partial \bar{p}_{i}(z)}{\partial z} \\
& -\frac{1}{\bar{n}(z)} \frac{\partial \widetilde{p}_{i}}{\partial z}-e \frac{\partial(\bar{\phi}(z)+\widetilde{\phi})}{\partial z}-M g \\
= & \frac{\tilde{n}}{\bar{n}^{2}(z)} \frac{\partial \bar{p}_{i}(z)}{\partial z}-\frac{1}{\bar{n}(z)} \frac{\partial \widetilde{p}_{i}}{\partial z}-e \frac{\partial \widetilde{\phi}}{\partial z} .
\end{aligned}
$$

We used

$$
-\frac{1}{\bar{n}(z)} \frac{\partial \bar{p}_{i}(z)}{\partial z}-e \frac{\partial \bar{\phi}(z)}{\partial z}-M g=0,
$$

due to the assumed isothermal steady-state condition. We took the ion dynamics to be adiabatic with $\gamma=C_{P} / C_{V}$ being the ratio of specific heats. It is readily demonstrated that (9) is consistent with the assumed isothermal condition for the ion component in steady state, giving $\bar{p}_{i}(z)=\bar{n}(z) T_{i}$.

The electron component is also here assumed to be a Boltzmann distribution at all times with constant temperature $T_{e}$, i.e., $n_{e}=n_{0} \exp \left(e \phi / T_{e}\right)$. We linearize this expression as

$$
\begin{aligned}
n_{e} \equiv \bar{n}+\tilde{n}_{e} & =n_{0} \exp \left(\frac{e \bar{\phi}+e \widetilde{\phi}}{T_{e}}\right) \\
& \approx n_{0} \exp \left(\frac{e \bar{\phi}}{T_{e}}\right)\left(1+\frac{e \widetilde{\phi}}{T_{e}}\right) .
\end{aligned}
$$

This result gives $\tilde{n}_{e}=\left(e \phi / T_{e}\right) n_{0} \exp \left(e \bar{\phi} / T_{e}\right)$, or $e \phi / T_{e}=\eta_{e}$.

We use $n=n_{0}\left(p / p_{0}\right)^{1 / \gamma}$ where $p=n T_{i}$ to obtain a dynamic equation for the ion temperature. This inserted into the equation of ion continuity gives after some simple manipulations the ion pressure equation,

$$
\left(\frac{\partial}{\partial t}+u_{z} \frac{\partial}{\partial z}\right) p=-\gamma p \frac{\partial}{\partial z} u_{z},
$$


where the consequences of compressibility appear explicitly by the right-hand side. The spatial derivative terms on the left side account for the convection of pressure perturbations.

Linearizing the ion pressure equation we have

$$
\frac{\partial \widetilde{p}_{i}}{\partial t}+u_{z} \frac{d \bar{p}_{i}}{d z}=-\gamma \bar{p}_{i} \frac{\partial}{\partial z} u_{z}
$$

Introducing the normalized quantity $\zeta \equiv \widetilde{p}_{i} / \bar{p}_{i}$ we find

$$
\frac{\partial \zeta}{\partial t}+u_{z} \frac{d \ln \bar{p}_{i}}{d z}=-\gamma \frac{\partial}{\partial z} u_{z}
$$

We use

$$
\frac{\partial \widetilde{p}_{i}}{\partial z} \equiv \frac{\partial \zeta \bar{p}_{i}}{\partial z}=\bar{p}_{i} \frac{\partial \zeta}{\partial z}+\zeta \frac{d \bar{p}_{i}}{d z},
$$

and with $\bar{p}_{i}=\bar{n} T_{i}$ find by the linearized ion momentum equation,

$$
M \frac{\partial u_{z}}{\partial t}=(\eta-\zeta) T_{i} \frac{d \ln \bar{n}(z)}{d z}-T_{i} \frac{\partial \zeta}{\partial z}-e \frac{\partial}{\partial z} \widetilde{\phi}
$$

\section{B. Dispersion: Poisson's equation}

With Boltzmann distributed electrons, Poisson's equation has the nonlinear form,

$$
\frac{\partial^{2} \phi}{\partial z^{2}}=\frac{e}{\varepsilon_{0}}\left(n_{e}-n_{i}\right)=\frac{e}{\varepsilon_{0}}\left(\bar{n} \exp \left(e \phi / T_{e}\right)-n_{i}\right) .
$$

With the present approximations, this equation is the only one where $T_{e}$ appears. Linearizing (12) we find

$$
\begin{aligned}
\frac{\partial^{2} \tilde{\phi}}{\partial z^{2}} & =\frac{e}{\varepsilon_{0}}\left(n_{0} \exp \left(\frac{e \bar{\phi}(z)}{T_{e}}\right) \frac{e \widetilde{\phi}}{T_{e}}-\widetilde{n}_{i}\right) \\
& =\frac{e}{\varepsilon_{0}}\left(\bar{n}(z) \frac{e \widetilde{\phi}}{T_{e}}-\widetilde{n}_{i}\right), \\
\frac{\partial^{2} e \tilde{\phi} / T_{e}}{\partial z^{2}} & =\frac{e^{2} \bar{n}(z)}{\varepsilon_{0} T_{e}}\left(\frac{e \tilde{\phi}}{T_{e}}-\eta\right) .
\end{aligned}
$$

The latter form contains the Debye length explicitly on the right-hand side. For the present problem we have $\lambda_{\mathrm{De}}=$ $\sqrt{\varepsilon_{0} T_{e} /\left(e^{2} \bar{n}(z)\right)}$. As $z \rightarrow \infty$ we have $\lambda_{\text {De }}(z) \rightarrow \infty$ and (13) shows that the assumption of quasineutrality will necessarily break down above some altitude at the arrival of any pulse characterized by some given length scale, even though the initial condition near $z=0$ is quasineutral.

The complete set of linear equation for the normalized quantities $\eta=\widetilde{n}_{i} / \bar{n}$ and $\zeta=\widetilde{p}_{i} / \bar{p}_{i}$ is

$$
\begin{gathered}
\frac{\partial \eta}{\partial t}-\frac{u_{z}}{L_{g}}+\frac{\partial u_{z}}{\partial z}=0, \\
\frac{\partial u_{z}}{\partial t}=-(\eta-\zeta) \frac{u_{T i}^{2}}{L_{g}}-u_{T i}^{2} \frac{\partial \zeta}{\partial z} \\
-\frac{e}{M} \frac{\partial \phi}{\partial z}, \\
\frac{\partial \zeta}{\partial t}-\frac{u_{z}}{L_{g}}=-\gamma \frac{\partial u_{z}}{\partial z}, \\
\frac{\partial^{2} \phi}{\partial z^{2}}=\frac{e \bar{n}}{\varepsilon_{0}}\left(\frac{e \phi}{T_{e}}-\eta\right) .
\end{gathered}
$$

We have $\gamma=5 / 3$ for adiabatic ion dynamics. Alternatively, $\gamma=1$ for isothermal dynamics and we have $\zeta=\eta$ there. Taking a plane test wave $\exp (-i(\omega t-k z))$ we find a dispersion relation in the form,

$$
\omega=\frac{\sqrt{k} \sqrt{k L_{g}+i}}{\sqrt{i L_{g}\left(k^{2} \lambda_{\mathrm{De}}^{2}+1\right)}} \sqrt{C_{s}{ }^{2}+\gamma u_{T i}{ }^{2}\left(k^{2} \lambda_{\mathrm{De}}^{2}+1\right)} .
$$

The result is local in the sense that we take $e^{2} \bar{n} / \varepsilon_{0} T_{e}$ constant [5].

Assume the ratio of the Debye length and the vertical length scale $\lambda_{D e} / L_{g} \sim \epsilon^{2}$, where $\epsilon$ is a small dimensionless expansion parameter. We now expand the dispersion relation in powers of $\epsilon$. To lowest order we get the nondispersive sound relation $\omega \approx k C_{s}$, where the sound speed $C_{s}=$ $\sqrt{\left(T_{e}+\gamma T_{i}\right) / M} \approx \sqrt{T_{e} / M}$ when $T_{e} \gg T_{i}$ as in our case. To the next order in $\epsilon$ we find the additional term,

$$
\frac{\left(T_{e} / M\right)\left(1+i k^{3} \lambda_{\mathrm{De}}^{2} L_{g}\right)+\gamma u_{T i}^{2}}{2 L_{g} C_{s}}=\frac{C_{s}}{2 L_{g}}+i \frac{k^{3}}{2} C_{s} \lambda_{\mathrm{De}}^{2},
$$

see also Fig. 1. We will use $C_{s} \approx \sqrt{T_{e} / M}$ in the following analysis. The linear differential equation for one of the plasma variables, say $u_{z}(z, t)$, is obtained by the replacements $\omega \rightarrow i \partial / \partial t$ and $k \rightarrow-i \partial / \partial z$.

\section{THE KORTEWEG-DE VRIES EQUATION}

By a standard reductive perturbation analysis we can obtain a modified Korteweg-de Vries (KdV) equation. Details of the method can be found in a monograph [17], and in particular also in the special issue on "Reductive Perturbation Method for Nonlinear Wave Propagation," Supplement of the Progress in Theoretical Physics (1974), Vol. 55, published by the Research Institute for Fundamental Physics and the Physical Society of Japan. In the present analysis we retain the lowest order correction in the dispersion relation originating from Poisson equation, i.e., deviations from quasineutrality. We assume that the ratio of the electron Debye length and the vertical length scale $\lambda_{\mathrm{De}} / L_{g} \sim \epsilon^{2}$ is of the same order as the fluid steepening nonlinearity in the expansion parameter. To lowest order in the small expansion parameter we therefore recover the linear nondispersive sound waves propagating in homogeneous plasmas. To next order we here have dispersion, nonlinearity, and the effects of the large-scale density gradient entering at the same level. We find a modified $\mathrm{KdV}$ equation in the form,

$$
\frac{\partial u_{z}}{\partial t}+\left(C_{s}+u_{z}\right) \frac{\partial u_{z}}{\partial z}+\frac{1}{2} C_{s} \lambda_{\operatorname{De}}^{2} \frac{\partial^{3} u_{z}}{\partial z^{3}}=\frac{g}{2 C_{s}} u_{z} .
$$

To lowest order (20) reproduces (4) in the limit of large $k_{z}$, i.e., for structures that are narrow in comparison with $L_{g}$. The coefficient to the dispersion term, $\partial^{3} u_{z} / \partial z^{3}$, contains the Debye length which varies slowly with $z$ due to the vertical density gradient. As long as a pulse moves a distance smaller than $L_{g}$ we can let $\lambda_{\text {De }}$ be constant and let the nonlinear pulse parameter variations be controlled predominantly by the varying amplitude. The term on the right-hand side of (20) gives rise to a growth of the velocity perturbation associated with a soliton or any other initial condition. The equation is here expressed for the space time varying velocity $u_{z}$, but to 


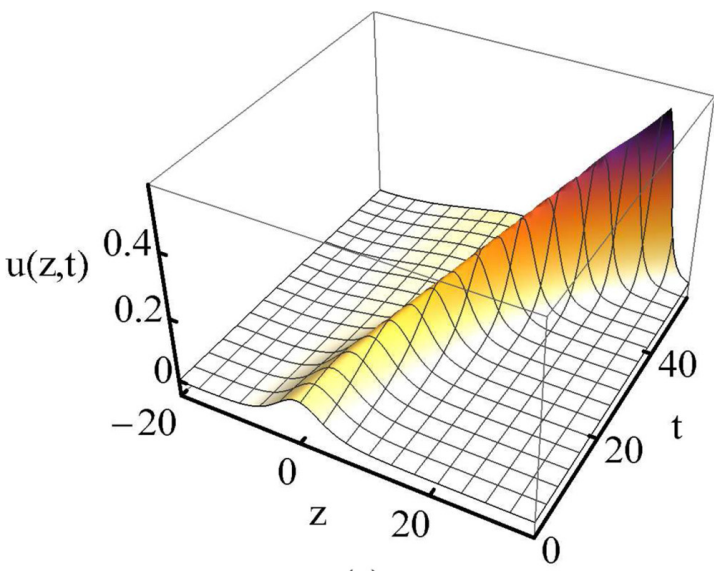

(a)

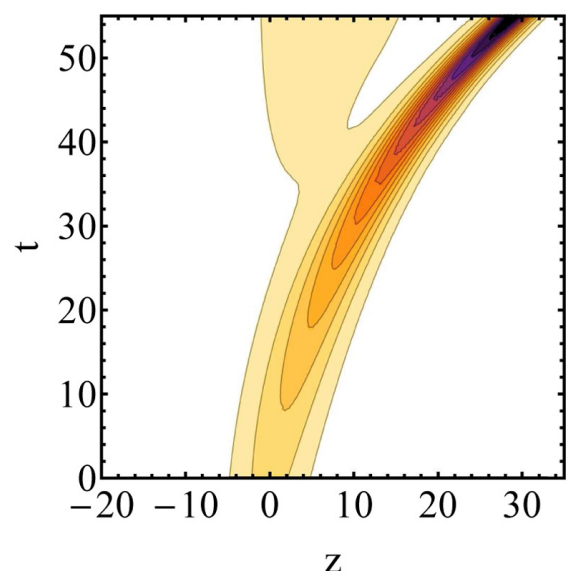

(b)

FIG. 3. Numerical solution of the modified KdV equation (20) in the normalized form $\partial_{t} u+6 u \partial_{x} u+\partial_{x x x} u=\gamma u$ are shown in (a) with the initial pulse shape being a soliton like (22) with amplitude $A_{0}=$ 0.1 and taking $\gamma=0.025$. The pulse is "speeding up" and becomes narrower as its amplitude increases due to the growth term on the right-hand side of (20). Note the formation of a "plateau" trailing the soliton. There is an analytical basis also for this result $[11,16]$. The figure refers to a frame of reference moving with the ion sound velocity. (b) A contour representation of (a).

lowest order we can use the relation $e \phi / T_{e} \approx u_{z} / C_{s}$ to establish an equation for the electrostatic potential $\phi$. Often the $\mathrm{KdV}$ equation is written in the frame moving with the sound velocity. Illustrative numerical solutions of (20) in this frame are shown in Fig. 3. This solution refers to the idealized case with the initial condition being an exact soliton solution which is usually considered in a perturbation analysis. In the absence of a density gradient it will propagate without deformation through the system. Note the formation of a plateau trailing the soliton for the inhomogeneous $\mathrm{KdV}$ equation. Ultimately also this plateau will breakup into a new small amplitude soliton as seen for large times in Fig. 3.

The KdV equation is an approximation to the set of dynamic equations, and the perturbation term on the righthand side of (20) also represents an approximation to the full modification induced by the plasma density gradient. We cannot expect an exact energy conservation by (20).
The interest in these growing pulse solutions is due to the possibility for soliton interactions with plasma particles, in particular acceleration of particles by a first-order Fermi acceleration [18]. If applied to ionospheric conditions, such types of wave particle interactions can contribute also to polar wind accelerations.

As well known, a KdV-type equation describes unidirectional propagation of pulses. We can formulate a slightly more general Boussinesq equation as shown in Appendix A. This equation can have interest in its own right, but will not be used here.

\section{SUMMARY FOR THE HOMOGENEOUS KdV EQUATION}

For later use we first summarize some relevant results for $\mathrm{KdV}$ solitons. The homogeneous KdV equation in the general form,

$$
\frac{\partial}{\partial t} u+\beta u \frac{\partial}{\partial z} u+\alpha \frac{\partial^{3}}{\partial z^{3}} u=0,
$$

has soliton solutions

$$
u=A \operatorname{sech}^{2}\left(\left(z-U_{s} t\right) \sqrt{A \beta / 12 \alpha}\right),
$$

where the soliton velocity scales linearly with amplitude as $U_{s}=A \beta / 3$. The soliton width $\Delta=\sqrt{12 \alpha / A \beta}$ scales inversely with the square root of the soliton amplitude. Large amplitude solitons are fast and narrow. By the inverse scattering transform $[19,20]$ it can be demonstrated that any compact initial perturbation will in time develop into one or more solitons followed by a low level of oscillations well described by the linearized version of the $\mathrm{KdV}$ equation.

For the present analysis it is implicitly assumed that the soliton is local in the sense that its width is smaller than the characteristic length scale, i.e., $\Delta \ll L_{g}$. The parameters here are $\alpha=\frac{1}{2} C_{s} \lambda_{\text {De }}^{2}$ and $\beta=1$ by (20). As an estimate we have the velocity amplitude related to the density perturbation as $A \approx C_{s} \delta n / n_{0}$. The requirement $\Delta \ll L_{g}$ then imposes the restriction $\sqrt{6 \lambda_{\mathrm{De}}^{2} n_{0} / \delta n} \ll 2 T / M g$ or $\delta n / n_{0} \gg$ $\frac{3}{2} \lambda_{\mathrm{De}}^{2} M^{2} g^{2} / T^{2}$, which can be reduced to the simpler expression $\delta n / n_{0} \gg \frac{3}{2}\left(\lambda_{\mathrm{De}} / L_{g}\right)^{2}$. This requirement has to be imposed on the excitation of the soliton and the results are valid as long as the inequality is fulfilled, where $n_{0}$ then refers to the plasma density at the soliton position.

An infinite number of conservation laws are associated with the homogeneous $\mathrm{KdV}$ equation. A few examples are [21]

$$
\begin{gathered}
I_{1} \equiv \int_{-\infty}^{\infty} u(z, t) d z, \\
I_{2} \equiv \int_{-\infty}^{\infty} \frac{1}{2} u^{2}(z, t) d z,
\end{gathered}
$$

etc., where $I_{2}$ in particular is often associated with the energy of a perturbation. We note here that this interpretation assumes homogeneous media. For the soliton solution (22) we find $I_{1}=4 \sqrt{3 A \alpha / \beta}$ and $I_{2}=4 A \sqrt{A \alpha /(3 \beta)}$. With an average position being $\int_{-\infty}^{\infty} z u(z, t) d z$ we find a pulse velocity to be $\int_{-\infty}^{\infty} z \partial u(z, t) / \partial t d z$. For a soliton solution we readily find the velocity to be $U_{s}$ as given before. The conservation laws (23) and (24) are valuable for a subsequent perturbation analysis. 


\section{SOLITON PERTURBATION ANALYSIS}

Korteweg-de Vries equations with perturbations have been studied in detail [16,22-26]. We follow the simplest analysis based on conservation laws [22]. Retaining the perturbation term on the right-hand side of (20) the conservation laws become

$$
\begin{aligned}
& \frac{d I_{1}}{d t}=\frac{g}{2 C_{s}} I_{1}, \\
& \frac{d I_{2}}{d t}=\frac{g}{C_{s}} I_{2},
\end{aligned}
$$

with solutions $I_{1}(t)=I_{1}(0) \exp \left(\frac{1}{2} t g / C_{s}\right) \quad$ and $\quad I_{2}(t)=$ $I_{2}(0) \exp \left(t g / C_{s}\right)$. Taking the initial perturbation to have a soliton shape we have $I_{1}(0)=4 \sqrt{3 A_{0} \alpha / \beta}$ and $I_{2}(0)=4 A_{0} \sqrt{A_{0} \alpha /(3 \beta)}$.

Starting the problem with a soliton solution we assume that it at all times relaxes to retain its soliton shape: For slow variations this assumption is justified by the inverse scattering transform. Since the soliton is a one-parameter solution to the $\mathrm{KdV}$-equation, we can at all times quantify its characteristics by its amplitude. Velocity and width follows from this amplitude. A small nonsoliton part, $u_{n s}$ as seen developing at late times in Fig. 3, is necessary to accommodate the difference between the entire solution $u_{z}(z, t)$ and the time evolving soliton part $u_{s}$. Since the nonsoliton part has a small amplitude it has a small velocity in the frame of reference moving with $C_{s}$ and it will be a "tail" following the soliton: We assume that the overlap between these two components of $u_{z}(z, t)$ is negligible implying $u_{s}(z, t) u_{n s}(z, t) \approx 0$. The plateau starts at $z \approx 0$ in the moving frame and ends at the soliton position in the moving frame $\langle z(t)\rangle=\int_{0}^{t} U_{s}(\tau) d \tau$ in terms of the soliton velocity $U_{s}(t)=A(t) \beta / 3$. We let the plateau be characterized by a spatially averaged amplitude $\xi(t)$, so that $I_{1}(t) \approx\langle z(t)\rangle \xi(t)+4 \sqrt{3 A(t) \alpha / \beta}$ and $I_{2}(t) \approx$ $\langle z(t)\rangle \xi^{2}(t)+4 A(t) \sqrt{A(t) \alpha /(3 \beta)}$. We will later need $I_{2}(t)$ to be expressed in terms of the local soliton amplitude $A(t)$.

Together with the first two conservation laws we have two equations for the two unknowns, $A(t)$ and $\xi(t)$, since the time varying soliton velocity and thereby $\langle z(t)\rangle$ are determined through the soliton amplitude $A(t)$. Assuming $\xi$ to represent a small correction, we ignore terms containing $\xi^{2}$. From the expression for $I_{2}(t)$ we then have

$$
A(t) \approx A(0) \exp \left(t \frac{2 g}{3 C_{s}}\right) .
$$

This exponential growth is consistent with the numerical results in Fig. 3. As the length of the plateau increases, it can itself break up into solitons. As a consequence a local density and thereby also a local potential minimum develops behind the soliton which can subsequently participate in the kinetic particle interactions.

The soliton position in the moving frame is found by

$$
\begin{aligned}
\langle z(t)\rangle & \approx \int_{0}^{t} U(\tau) d \tau=\frac{A(0) \beta}{3} \int_{0}^{t} \exp \left(\tau \frac{2 g}{3 C_{s}}\right) d \tau \\
& =\frac{A(0) C_{s} \beta}{2 g}\left(\exp \left(t \frac{2 g}{3 C_{s}}\right)-1\right),
\end{aligned}
$$

where we for the present case have $\beta=1$. To transform to the fixed frame we have to add $t C_{s}$.

Using the results for $I_{2}(t)$ we can obtain an approximate expression for an equivalent of the kinetic energy of the system as

$$
\mathcal{E}_{k} \approx M n_{0} \exp \left(-\frac{t g}{C_{s}}\right) I_{2}(t)=\text { constant },
$$

at any time $t$, recalling that this expression is meaningful only in the rest frame. The integration in $I_{2}$ entering (28) implicitly assumes $\Delta \ll L_{g}$, i.e., the soliton is local. We approximated the soliton position as $z \approx t C_{s}$ in $\bar{n}(z)=n_{0} \exp \left(-z g / C_{s}^{2}\right)$. For large times we find $\mathcal{E}_{k} \rightarrow$ constant to the lowest approximation. The contribution of the electrons to the total energy can be determined the same way.

Given $A=A(t)$ we can determine the average amplitude of the nonsoliton part $\xi(t)$ by the expression for $I_{1}(t)$. After some algebra we find

$$
\begin{aligned}
\xi(t) & =\frac{8 g \sqrt{3 \alpha}}{C_{s} \beta \sqrt{A(0) \beta}} \frac{\exp \left(\frac{t g}{2 C_{s}}\right)-\exp \left(\frac{t g}{3 C_{s}}\right)}{\exp \left(\frac{t 2 g}{3 C_{s}}\right)-1}, \\
\lim _{t \rightarrow \infty} \xi(t) & =\frac{8 g \sqrt{3 \alpha}}{C_{s} \beta \sqrt{A(0) \beta}} \exp \left(-\frac{t g}{6 C_{s}}\right) .
\end{aligned}
$$

At large times the soliton amplitude is exponentially large and so is its velocity unless the growth is arrested. Asymptotically, the nonsoliton tail is stretched out to have a small amplitude. A large initial amplitude $A(0)$ has the same effect.

The derivation of the KdV equation is accurate only to second order in the expansion parameter. For unstable conditions, the equation and its soliton solution will eventually become inaccurate when the higher order terms become important. For plasma solitons the growth will, however, eventually be arrested by the interaction of the solitary pulse with plasma particles, in our case the ion component.

For the entire energy budget we have in principle to include both the soliton and the nonsoliton parts. For interaction with particles, we need to be concerned only with the soliton part since it has the dominant amplitude.

\section{INTERACTION BETWEEN ION ACOUSTIC SOLITONS AND IONS}

The foregoing analysis emphasizes fluid models. The problem of plasma wave propagation in gravitational field in a horizontally striated plasma environment has previously $[27,28]$ been studied by linear kinetic models, including effects of Landau damping. The time interval with linear Landau damping is, however, of minor relevance for the problem when the nonlinear soliton evolution is considered. To see this we introduce a few relevant time scales: (i) a linear pulse time scale $\tau_{L}=\Delta / C_{S}$, which corresponds to the linear sound dispersion relation; (ii) we have a nonlinear soliton time scale $\tau_{S}$ which accounts for the time it takes a soliton to move its own width due to the nonlinear velocity correction [25,29], i.e., the motion in the frame moving with the sound speed $C_{S}$, giving $\tau_{S}=\Delta / U_{S}$ where $\tau_{S} \gg \tau_{L}$. In classifying the interaction between particles and wave pulses we have a time of linear or resonant interaction $\tau_{R}=\Delta / \sqrt{2 e \Psi / M} \sim \tau_{S}$ where 
$\Psi$ is the peak value of the electrostatic potential for the soliton. The velocity interval for resonant wave-particle interaction is $\left[C_{s}+U_{s}-\sqrt{2 e \Psi / M} ; C_{s}+U_{s}+\sqrt{2 e \Psi / M}\right]$ specifying the role of the soliton amplitude. The linear Landau damping is associated with transiting particles [30] while we here consider reflected particles.

We thus distinguish two parameter ranges: (i) times $t<$ $\tau_{R}$ where linear Landau damping dominates and soliton dynamics is of minor importance; (ii) times $t>\tau_{R} \sim \tau_{S}$ where soliton dynamics is important and the interaction between the nonlinear sound pulse and particles is (in our case) dominated by reflected ions.

To describe the propagation of weakly nonlinear sound waves in a kinetic model, several authors [31-34] have proposed a modified $\mathrm{KdV}$-equation in the form,

$$
\frac{\partial}{\partial t} \phi+\beta \phi \frac{\partial}{\partial z} \phi+\alpha \frac{\partial^{3}}{\partial z^{3}} \phi+\frac{s}{\pi} \mathcal{P} \int_{-\infty}^{\infty} \frac{1}{z-z^{\prime}} \frac{\partial \phi}{\partial z^{\prime}} d z^{\prime}=0,
$$

with $\mathcal{P}$ denoting the principal value of the integral and $\beta, \alpha$, and $s$ being suitably defined constants. The nonlocal integral term accounts for the linear Landau damping here and in a number of related studies [35-37], and the equation is thus valid for the time range (i) discussed before. In this time interval the solitons properties had little time to be manifested in any significant manner. The applicability of (29) is limited as far as the nonlinear soliton dynamics are concerned, although the equation as such has received attention in the past.

Many of the foregoing results had applications for general $\mathrm{KdV}$ equations. The present problem concerns acceleration of plasma particles by solitons propagating in gravitational plasmas with a vertical density gradient. For this case we have $\beta=1$ in (21) while $\alpha=\frac{1}{2} C_{s} \lambda_{\text {De }}^{2}$; see also (20). The simple model used here assumes electrons to be an isothermally Boltzmann distributed fluid at all time, with electron inertia effects ignored. The only plasma particles we need to be concerned with are the ions.

Given a soliton with velocity amplitude $A(t)$ we have the corresponding peak potential amplitude to be $\Psi(t)=$ $A(t)\left(T_{e} / e\right) / C_{s}$. The velocity interval for resonant ion interaction has then the form $\left[C_{s}\left(1+\frac{1}{3} e \Psi(t) / T_{e}\right)-U_{R} ; C_{s}(1+\right.$ $\left.\left.\frac{1}{3} e \Psi(t) / T_{e}\right)+U_{R}\right]$. Particles slower than $C_{s}\left(1+\frac{1}{3} e \Psi(t) / T_{e}\right)$ give up energy, while faster particles receive energy from the moving soliton. For the ions overtaking the soliton there would be a slight correction due to the plateau, but this will be ignored here. We here introduced $C_{s}\left(1+\frac{1}{3} e \Psi(t) / T_{e}\right)$ for the rest frame soliton velocity so that $U_{s}=\frac{1}{3} e C_{s} \Psi(t) / T_{e}$. We find that $\tau_{S} / \tau_{R} \sim C_{S} / \sqrt{2 e \Psi / M} \gg 1$. When the soliton dynamics is important, the linear Landau damping is of minor concern. The important soliton-particle interaction is caused by reflected particles, which is a nonlinear effect.

The following discussion will be based on energy conservation between a system consisting of a soliton and plasma particles, noting that ion sound waves have an approximate equipartition between ion and electron energy for long wavelengths [11]. The electric field energy is small in comparison. To be specific we have the change in energy density caused by a sound perturbation in the form,

$$
\Delta W=\frac{1}{2} M n_{0} \widetilde{u}^{2}+\frac{1}{2} \varepsilon_{0}(\nabla \phi)^{2}+\frac{1}{2} n_{0} \kappa T_{e}\left(\frac{e \phi}{\kappa T_{e}}\right)^{2},
$$

to lowest order, where it was assumed that $T_{i} \approx 0$.

In the following we will use the capital letter $U$ denoting the $z$ component of one ion as distinguished from a fluid velocity. The ion + electron energy of an ion acoustic soliton in a gravitational field is

$$
\mathcal{E} \approx 4 \sqrt{\frac{2}{3}}\left(\frac{e \Psi(t)}{T_{e}}\right)^{3 / 2} n_{0} \exp \left(-\frac{t g}{C_{s}}\right) T_{e} \lambda_{\mathrm{De}} ;
$$

see also (28). With $\Psi(t) \sim \exp \left(t 2 g / 3 C_{s}\right)$ [see (27)], we find $\mathcal{E} \sim$ constant in the absence of particle interactions or other additional perturbations.

Upon interaction with a soliton moving at velocity $U_{s}$, an ion changes its initial velocity $U$ by the amount $2 U_{s}$. The energy gain by such an interacting (i.e., resonant) ion is $2 M U_{s}\left(U_{s}-U\right)$, assuming the interaction to be perfectly elastic. A negative ion velocity (counterpropagating particles) gives net particle energy gain, positive ion velocities (overtaking collisions) give particle energy loss. The flux of these interacting ions is at some vertical position $z$ given as $\left|u-U_{s}\right| \bar{n}(z) f_{0}(u)$, where $f_{0}(u)$ is the normalized background ion velocity distribution function, $\int_{-\infty}^{\infty} f_{0}(u) d u=1$. Consequently at a time where the soliton has arrived (approximately) at a position $z=C_{s} t$, we can write the energy gain by resonant ions per unit time as

$$
\begin{aligned}
\frac{d \mathcal{E}_{\text {res }}}{d t}= & 2 M U_{s} n_{0} \exp \left(-\frac{t g}{C_{s}}\right) \\
& \times \int_{U_{\min }}^{U_{\max }}\left(U_{s}-U\right)\left|U-U_{s}\right| f_{0}(U) d U .
\end{aligned}
$$

The integration limits are $\left(U_{\min } ; U_{\max }\right)=$ $\left(U_{s}-\sqrt{2 e \Psi(t) / M} ; U_{s}+\sqrt{2 e \Psi(t) / M}\right)$.

We now equate this change in energy per time unit with the negative time derivative of the change in soliton energy obtained from (31). The foregoing arguments assume that the soliton amplitude $\Psi(t)$ changes only little during the transit time of an ion.

The foregoing analysis refers to one soliton interacting with particles. For larger soliton densities, solitons can interact due to mutually reflected particles [38]. A statistical analysis of such many-soliton cases has also been suggested [39].

We have found the energy gained or lost by ions accelerated or decelerated by a soliton. By energy conservation we know that this energy is lost or gained by the soliton. All soliton parameters can be expressed by the maximum soliton amplitude $\Psi(t)$ for the KdV soliton discussed here. Since a relation between the soliton parameter and the soliton energy is known we can obtain an equation for $\Psi(t)$. The rate of change of soliton energy for varying $\Psi(t)$ is

$$
\begin{aligned}
\frac{d \mathcal{E}}{d t}= & 4 \sqrt{\frac{2}{3}} \sqrt{\frac{e \Psi(t)}{T_{e}}} n_{0} \exp \left(-\frac{t g}{C_{s}}\right) T_{e} \lambda_{D e} \\
& \times\left(\frac{e}{T_{e}} \frac{d \Psi}{d t}-\frac{g}{C_{s}}\left(\frac{e \Psi(t)}{T_{e}}\right)\right) .
\end{aligned}
$$




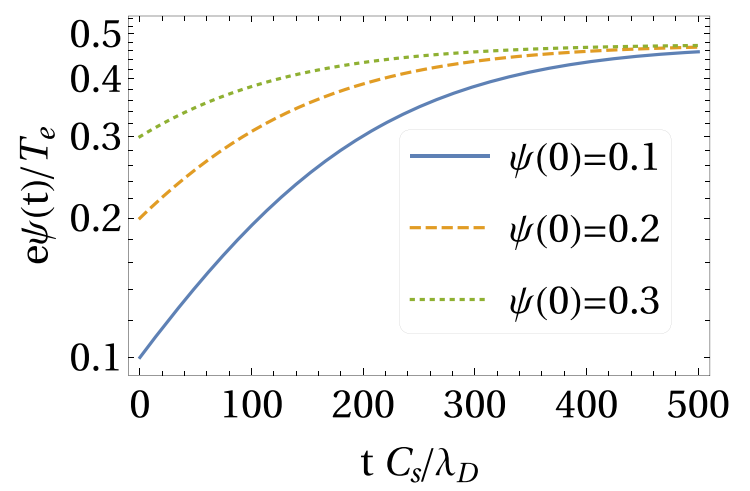

FIG. 4. Illustration of the normalized amplitude variation of an ion acoustic soliton as described by (34) for three different initial soliton amplitudes: $\Psi(0)=0.1,0.2$, and 0.3 . The figure uses normalized units, with a logarithmic vertical axis and $C_{s} \equiv \sqrt{T_{e} / M}$. We have here $T_{e} / T_{i}=10$ and a dimensionless "gravity parameter" $g \lambda_{D e} / C_{s}^{2}=0.01$. Less interesting solutions with larger initial amplitude, $\Psi(0)>0.5$ for the present parameters, damp out to reach the same asymptotic level as shown in the figure for the other amplitudes.

Equating (33) and (32) we note that an exponential factor cancels, and obtain after some algebra,

$$
\begin{aligned}
\frac{d}{d t} \frac{e \Psi(t)}{T_{e}}= & \frac{g}{C_{s}} \frac{e \Psi(t)}{T_{e}} \\
& +\frac{1}{3} \sqrt{\frac{3}{2}} \sqrt{\frac{T_{e}}{e \Psi(t)} \frac{M U_{s}}{T_{e} \lambda_{D e}} G\left(U_{s}, \Psi(t)\right),}
\end{aligned}
$$

with

$$
\begin{aligned}
G\left(U_{s}, \Psi(t)\right)= & \int_{U_{s}}^{U_{s}+\sqrt{2 e \Psi(t) / M}}\left(u-U_{s}\right)^{2} f_{0}(u) d u \\
& +\int_{U_{s}}^{U_{s}-\sqrt{2 e \Psi(t) / M}}\left(u-U_{s}\right)^{2} f_{0}(u) d u,
\end{aligned}
$$

recalling here that $U_{s}$ depends also on $\Psi(t)$, in general. For Maxwellian distributions, we can express $G\left(U_{s}, \Psi(t)\right)$ in terms of error functions. A numerical solution of (34) is shown in Fig. 4 assuming a Maxwellian distribution for $f_{0}(u)$. We find that a soliton with small initial amplitude has its peak potential amplitude increasing to maintain energy flux conservation for pulses propagating in an inhomogeneous system in the direction of decreasing density. At some time its amplitude is sufficiently large to have it interacting significantly with the ions. The growth is then arrested, eventually to reach a saturated level. The saturation level $e \Psi / T_{e} \approx 1 / 2$ found in Fig. 4 corresponds to $n(z) \approx n_{0}(z) / 2$. This value will, however, be found at large $z$ values where the ambient density $n_{0}$ is small, i.e., the initial perturbation at $z=0$ will be small. The results obtained by a local KdV equation will only serve as estimates in this saturation limit.

The saturation level and the time evolution in general depends on the electron-ion temperature ratio $T_{e} / T_{i}$ as well as $g / C_{s}$. If $T_{e} / T_{i}$ is reduced, the ion sound speed becomes closer to the ion thermal velocity and the soliton-particle interaction becomes stronger giving a lower saturation level. The asymptotic saturation level for the peak soliton potential does not in general have any simple analytical expression.
For the net soliton energy we have $\mathcal{E}(t \rightarrow \infty) \rightarrow 0$ when the soliton-particle interaction is taken into account for a stable plasma, e.g., a Maxwellian. The net kinetic energy gained by the particles equals the initial soliton energy. The density gradient acts as a "catalyst" mediating the energy transfer.

\section{A. Analytical approximations}

In order to obtain some quantitative results [29], we make a series expansion of $G\left(U_{s}, \Psi(t)\right)$ in (34), where we let the soliton velocity be a constant $U_{s} \approx C_{s}$ since the correction varies only with $\Psi(t)$ which was assumed to be small anyhow. The series are lengthy, and are given in Appendix A. Using these series, we can write the relation (34) to lowest order as

$$
\frac{d}{d t} \frac{e \Psi(t)}{T_{e}}=\frac{g}{C_{s}} \frac{e \Psi(t)}{T_{e}}+\sqrt{\frac{2}{3}} \frac{C_{s}^{3}}{\lambda_{\mathrm{De}}}\left(\frac{e \Psi(t)}{T_{e}}\right)^{3 / 2} f_{0}^{(1)}\left(C_{s}\right),
$$

which can be integrated to give

$$
\begin{aligned}
\frac{e \Psi(t)}{T_{e}}= & \left(\frac{g}{C_{s}}\right)^{2} \frac{e \Psi(0)}{T_{e}}\left(\left(\frac{g}{C_{s}}-v \sqrt{\frac{e \Psi(0)}{T_{e}}}\right)\right. \\
& \left.\times \exp \left(-\frac{t g}{2 C_{s}}\right)+v \sqrt{\frac{e \Psi(0)}{T_{e}}}\right)^{-2},
\end{aligned}
$$

where the damping constant is

$$
v=-\sqrt{\frac{2}{3}} \frac{C_{s}^{3}}{\lambda_{D e}} f_{0}^{(1)}\left(C_{s}\right) .
$$

When $f_{0}(u)$ is a Maxwellian, for instance, we have $f_{0}^{(1)}\left(C_{s}\right)<$ 0 giving $v>0$, and the soliton amplitude reaches an asymptotic saturation level

$$
\frac{e \Psi(\infty)}{T_{e}}=\left(\frac{g}{v C_{s}}\right)^{2} \equiv\left(\frac{\Omega_{p i}}{v} \frac{\lambda_{D i}}{L_{g}}\right)^{2},
$$

in terms of a reference density giving $\lambda_{D i}$ and $\Omega_{p i}$, independent of the initial value $\Psi(0)$. A characteristic time to reach an amplitude close to the saturation level is $t_{K} \approx$ $\left(v e \Psi(0) / T_{e}\right)^{-1} \sqrt{e \Psi(\infty) / T_{e}}$ and the corresponding propagation distance is approximately $C_{s} t_{K}$. These values are approximate: In the case where $\Psi(0)$ is close to $\Psi(\infty)$, the saturation is nearly instantaneous. We will usually be interested in conditions with $\Psi(0)<\Psi(\infty)$.

For a linearly unstable plasma where $f_{0}^{(1)}\left(C_{s}\right)>0$, giving $v<0$, we can find an "explosive" condition by (36) where $\Psi(t)$ can be diverging within a finite time $\tau_{c}$ given implicitly by

$$
\left(\frac{g}{C_{s}}-v \sqrt{\frac{e \Psi(0)}{T_{e}}}\right) \exp \left(-\frac{\tau_{c} g}{2 C_{s}}\right)=-v \sqrt{\frac{e \Psi(0)}{T_{e}}} .
$$

Such a "bump-on-tail" condition for the net ion velocity distribution can, for instance, be realized by an accelerated lighter ion component constituting the polar wind mentioned before. We recall, though, that these particles will have a 
steadily increasing vertical velocity and are thus not forming a classical bump-on-tail distribution.

Unfortunately, the compact result (36) has limited applicability [29]. This limitation can be illustrated by considering the next correction term in the series expansion in $G\left(U_{s}, \Psi(t)\right)$; see Appendix B. In this case we have

$$
\begin{aligned}
G\left(U_{s}, \Psi(t)\right) \approx & 2\left(\frac{e \Psi(t)}{M}\right)^{2} f_{0}^{(1)}\left(U_{s}\right) \\
& \times\left(1+\frac{1}{9} \frac{f_{0}^{(3)}\left(U_{s}\right)}{f_{0}^{(1)}\left(U_{s}\right)} \frac{2 e \Psi(t)}{M}\right) .
\end{aligned}
$$

For an order of magnitude estimate we can use a Maxwellian ion velocity distribution, $f_{0}(u)=$ $(2 \pi \sigma)^{-1 / 2} \exp \left(-u^{2} / 2 \sigma\right)$, with $\sigma \equiv T_{i} / M \ll C_{s}^{2}$. For the last correction term in the parenthesis to be small we require $\left(C_{s}^{2} / \sigma\right)\left(e \phi_{0} / T_{i}\right) \ll 5$, which is only marginally realistic in natural conditions (i.e., realistic sound speeds, velocity, distributions, etc.), when we at the same time require that the nonlinearities should be manifested in a reasonable time, i.e., that the soliton time should be moderate. It is most likely that (34) has to be solved numerically for realistic and relevant cases as in Fig. 4. We find that the saturation level $e \Psi(\infty) / T_{e}$ found by (36) to be an overestimate, in general.

\section{NUMERICAL SIMULATION RESULTS}

Our hybrid code with kinetic ions and mass-less isothermally Boltzmann distributed electrons assumes $n_{e}=$ $n_{0} \exp \left(e \phi / T_{e}\right)$ from the outset, implying that Poisson's equation becomes nonlinear in the present problem. This is a nonstandard element in the code. The ion component responds to the collective electric fields and to an imposed constant vertical gravitational field. The numerical simulation results allow for deviations from quasineutrality since Poisson's equation is explicitly included. The initial conditions can be chosen to have characteristic scale lengths much larger than $\lambda_{\text {De }}$ so that quasineutrality can be assumed, but at later times we can find smaller scales to develop and deviations from quasineutrality can become important. In this limit (12) will be relevant, and the expression is implemented in our particle-in-cell (PIC) code. The electrostatic code is used here in $2 \mathrm{R} 3 \mathrm{~V}$ mode, or $2.5 \mathrm{D}$, i.e., with two spatial dimensions while the velocities resolve three velocity dimensions in order to account for the particle motions in magnetic fields. The boundary conditions in the direction $\perp \mathbf{B}$ are periodic, while the upper and lower boundaries have Neumann conditions. Details of the code are described elsewhere $[13,40]$.

Most studies of KdV solitons are based on models in strictly one spatial dimension. To make the numerical analysis somewhat more physically relevant we consider a twodimensional magnetized system. A generalization to a fully three-dimensional system will in our case not bring any new features to the problem. The basic plasma parameters are chosen to be consistent with the assumptions of the model, i.e., $\Omega_{c i}>\Omega_{p i}$. Assuming an enhanced electron temperature in a central magnetic flux tube we can also here derive a $\mathrm{KdV}$ equation for a lowest order radial eigenmode. Such a "channel" with high electron temperatures is physically realistic and is often found in, for instance, laboratory plasmas [41], discharge plasmas and laser produced plasmas $[42,43]$, ionospheric heating experiments $[44,45]$, as well as in linearly unstable ionospheric plasma conditions [46]. One consequence of such a temperature channel is to provide a natural localization of the pulses in the direction perpendicular to $\mathbf{B}$; otherwise the scale size in that direction would be given by the initial conditions. A temperature gradient $\perp \mathbf{B}$ can in principle give rise to unstable electrostatic waves propagating in the direction perpendicular to both $\mathbf{B}$ and $\nabla_{\perp} T_{e}$ [47]. In a collisionless plasma these waves usually have a small growth rate and they have no relevance here. They will not be generated in the spatially two-dimensional numerical simulations where these waves would have to propagate in the direction perpendicular to the simulation plane. The present analysis is mathematically also related to studies of weakly nonlinear electrostatic Trivelpiece-Gould modes in a magnetized plasma waveguide $[37,48]$. Details of the analytical model in terms of eigenmodes as used also here are given elsewhere [13]. The basic analysis gives an equation for "simple waves" [49], which is subsequently generalized by introducing dispersion and the effect of gravity to give a modified Korteweg-de Vries equation.

Numerical simulations of gravitational systems are demanding by requiring an excessive number of simulation particles. This is so because of the large densities accumulating at lower altitudes, while only relatively few particles are left at the higher altitudes where the nonlinear features are manifested. The present simulations use $23 \times 10^{6}$ particles (approximately $10^{3}$ particles per $\lambda_{\mathrm{Di}}^{2}$ in terms of the reference length scale $\lambda_{\mathrm{Di}}$ ), where this number fluctuates slightly over time due to particle loss and injection at the lower boundary. This number of macroparticles is as for all PIC simulations not sufficient for realistic or even semirealistic plasma conditions, but the results can be used for a qualitative comparison with analytical results. The simulations have $\lambda_{\mathrm{Di}} \ll L_{g}$ but the ratio of the two length scales is much smaller than the ratio found in nature. The reference length scale $\lambda_{\mathrm{Di}}$ is found by using the average density in the simulation domain as mentioned before.

Results from numerical simulations are shown in Figs. 510. The figures show the simulation domain of $95 \times 250 \lambda_{\mathrm{Di}}^{2}$. In order to improve the signal-to-noise ratio in Fig. 5, we averaged four results from simulations with different seeding of the random number generators distributing the simulation particles in the $2 \mathrm{R} 3 \mathrm{~V}$ phase space. In Fig. 5 we show two results without gravity. One case has a temperature ratio of $T_{e} / T_{i}=10$, and a second reference case has a ratio of $T_{e} / T_{i}=$ 15. In the first case we observe the ion Landau damping, which is strongly reduced in the second case due to the larger ion sound speed $C_{s}=\sqrt{\left(T_{e}+\gamma T_{i}\right) / M}$. The solitons are shown at the same times, and the difference in their basic velocity is noticeable. The nonlinear velocity correction is small in comparison. The case including a gravitational acceleration is shown in Fig. 6.

The peak value of the soliton amplitude variations are shown in Fig. 7. We note in particular that this variation is exponential only for a restricted initial time interval, even for the case without gravitational forces, $G=0$.

For the gravitational case, $G \neq 0$, we find an amplitude increase as predicted by the simple model. Eventually the 

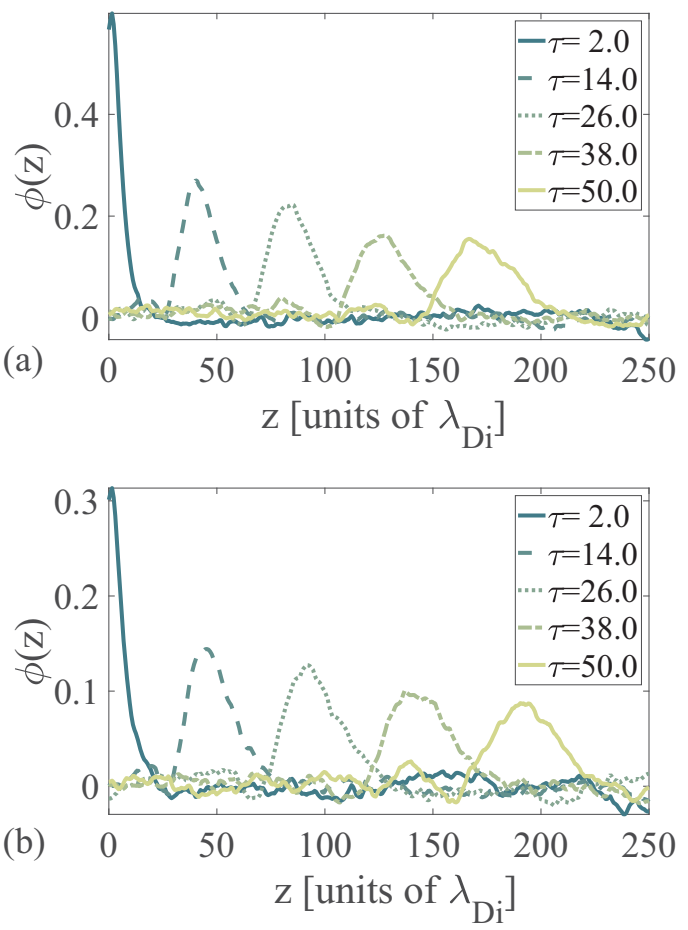

FIG. 5. Spatial variations of propagating solitons taken at selected time steps for the reference case with no gravitational field, $G=0$. We have $T_{e} / T_{i}=10$ in the top panel (a) and $T_{e} / T_{i}=15$ in the bottom panel (b), respectively. The damping is due to ion Landau damping, which is strongly reduced by the increased temperature ratio in the second case. The externally imposed excitation amplitudes are 0.25 and 0.1 for the two cases. The first narrow pulse on the figure is a part of the initial excitation. The difference in propagation velocity is due to the change in the sound speed.

soliton amplitude reaches a level where it interacts strongly with the particles and find an amplitude saturation for large times. We note the formation of a "forerunner" or precursor in front of the soliton for increasing times; see Fig. 6, for

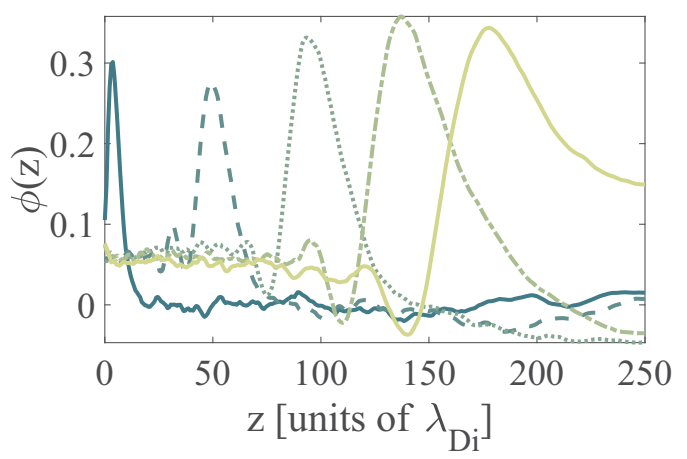

FIG. 6. Spatial variations of propagating solitons taken at selected time steps $\tau=2,14,26,38$, and $50 \Omega_{p i}^{-1}$, with $G=0.5$ in normalized units. We have $T_{e} / T_{i}=15$. Comparing with Fig. 5 we note an initially increasing amplitude due the growth induced by the plasma density gradient in the gravitational field. The first narrow pulse at $\tau=2 \Omega_{p i}^{-1}$ on the figure is also here a part of the initial excitation.

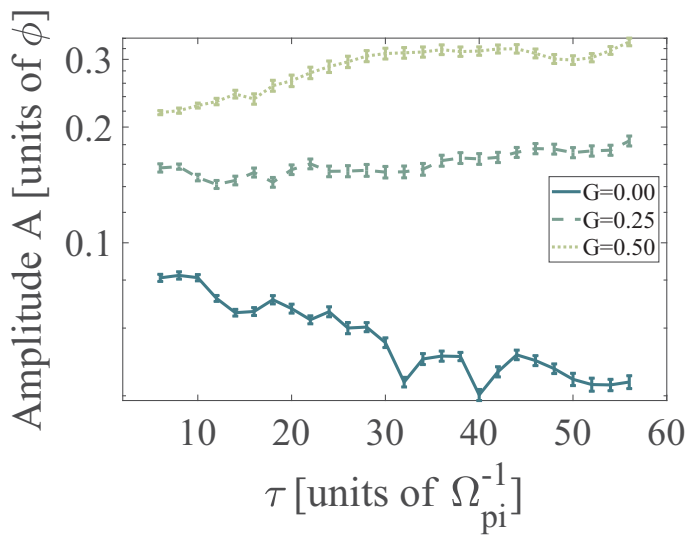

FIG. 7. Time evolution of the peak value of the soliton potential amplitude $A$ in computational units, together with error estimates obtained from fitting the electric potential $\phi(z)$ at times $\tau_{i}$ of the simulation to the three-parameter $\left(A, z_{0} \equiv U_{s} t, \Delta\right) \mathrm{KdV}$-soliton function given in (22). Note that the scale is logarithmic on the vertical axis. For the largest value of the gravitational acceleration $G=0.5$, in computational units, we have an initial time interval with a near exponential growth (seen as linear in the logarithmic scale). The ultimate saturation is due to ions reflected by the large amplitude sound pulse. Also shown is the time evolution for $G=0.25$ and $G=0$. We have $T_{e}=15 T_{i}$ for all cases.

instance. This is caused by the ions reflected and energized by the propagating soliton.

The soliton velocity as given in Fig. 8 is nearly constant, corresponding to the ion sound speed for the given conditions. Some "spikes" for the case with $G=0$ are due to inaccuracies in the numerical fitting procedures. The nonlinear velocity correction is small.

The variation of the soliton width is shown in Fig. 9. For the case with $G=0$ we find that the amplitude-width scaling predicted by the $\mathrm{KdV}$ equation is qualitatively correct.

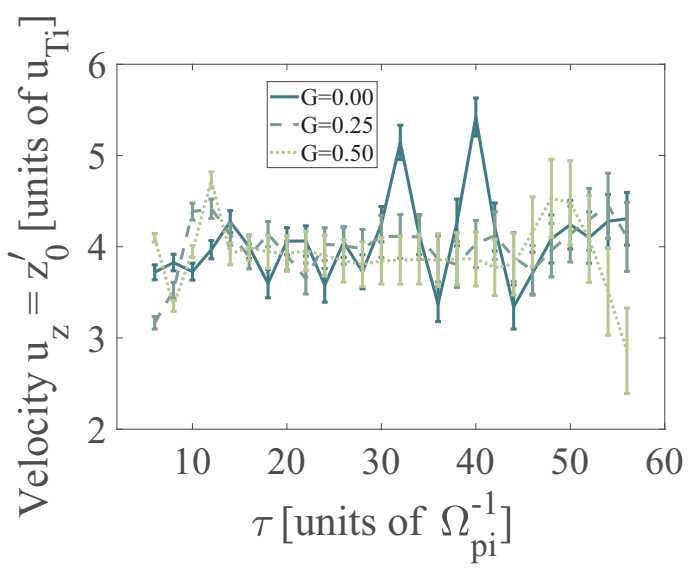

FIG. 8. Time evolution of the soliton velocity shown in units of ion thermal velocity together with error estimates. The velocity and error estimates are obtained by evaluating $z_{0}^{\prime}=d z_{0} / d \tau$ using a central finite difference of the discrete positions $z_{0 i}$ of the soliton maximum. The positions $z_{0 i}$ are obtained from the parametric fit of the electric potential $\phi(z)$ at times $\tau_{i}$ of the simulation described in Fig. 7. 


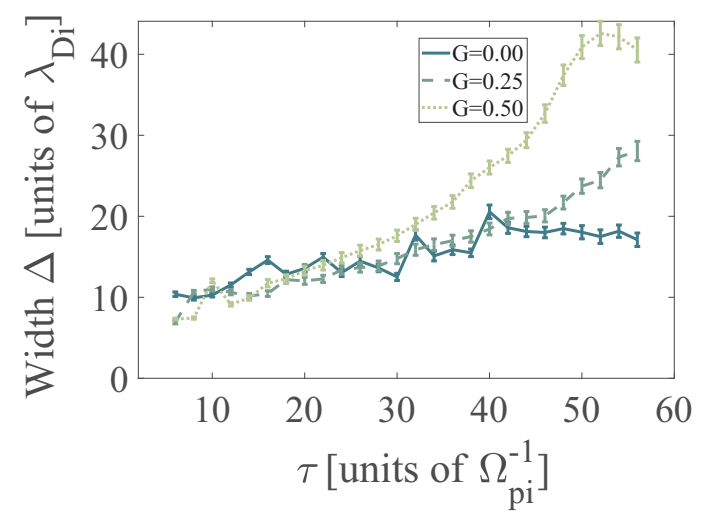

FIG. 9. Time evolution of the soliton width $\Delta$, measured in units of the reference ion Debye length, together with error estimates, obtained from the parametric fit of the electric potential $\phi(z)$ at times $\tau_{i}$ of the simulation described in Fig. 7.

When $G \neq 0$ we do not find this agreement. Most likely this disagreement is caused by the uncertainty in defining a proper soliton width when we have a precursor in the form of particles (in our case ions) reflected by the soliton.

The full configuration and phase space information is given in Fig. 10 for a late time $\tau=50 \Omega_{p i}$ in the evolution. The bulk

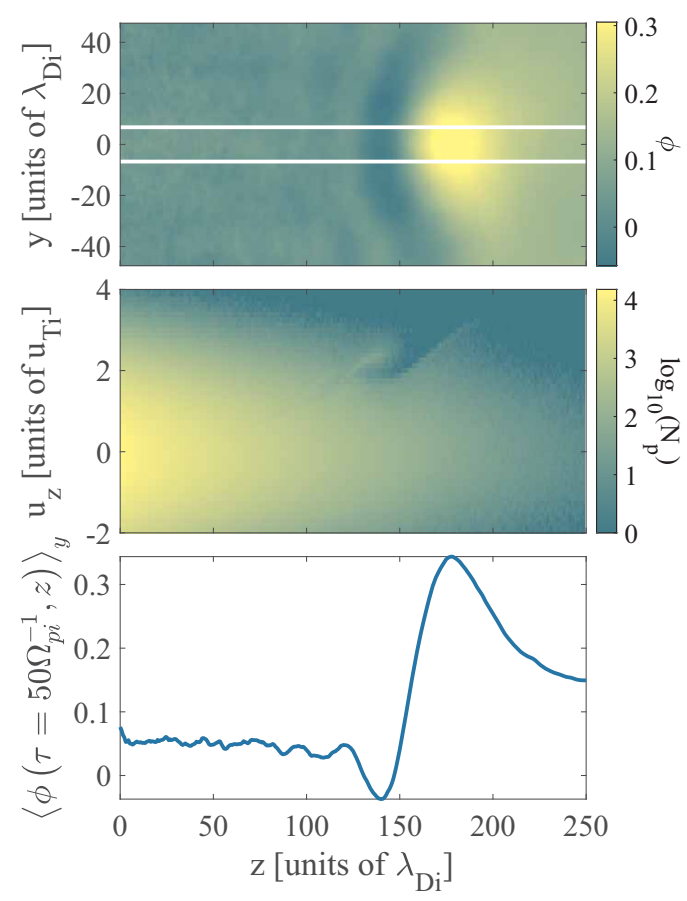

FIG. 10. (Upper panel) Color coded variation of the soliton in configuration space with linear scale of the electric potential $\phi$. (Middle panel) Phase space variation of the same structure with a logarithmic color scale, averaged over the central "channel" located between the white horizontal lines shown in the upper panel. (Lower panel) Corresponding potential variation along the $z$ axis, also averaged over the central part of the plasma column. All panels are for the time of simulation, $\tau=50 \Omega_{p i}^{-1}$. The gravitational acceleration points towards the negative direction of the $z$ axis while the magnetic field towards the positive direction. plasma density increases when moving from large $z$ towards $z=0$ consistent with a balance between the gravitational and plasma pressure forces as discussed in obtaining (7), for instance.

In front of the soliton we note the population of reflected ions: Visually, it appears similar to the "snow plow" effect found in front of shocks propagating in, for instance, coaxial plasma accelerators $[50,51]$. The solitary pulse is excited in the central part of the plasma (between the two white lines in the top figure). The boundary conditions for the electric field makes the pulse spread in the $y$ direction across magnetic field lines into the surrounding plasma where $T_{e}=T_{i}$.

The localized density depletion forming behind the soliton gives a potential well that can trap particles to form a phase space vortex there. In Fig. 10 we find the formation of such a phase space vortex behind the solitary form. These vortexlike structures have been found in controlled laboratory experiments first in electron phase space [52] and then also in ion phase space [53]; see also a summary [40]. In their basic form, phase space vortices are spatially one-dimensional [54], but can be found in a three-dimensional form in magnetized plasmas [55]. These structures are also influenced by the inhomogeneous plasma conditions [56-58] but they do not contribute to the particle acceleration. The phase space vortices are purely kinetic phenomena and usually propagating with moderate velocities, in our case near the ion thermal velocity while the soliton propagates slightly faster than the ion sound speed. The distance between vortex and soliton will consequently be steadily increasing once the vortex is fully developed.

A number of observations can be made on the basis of the simulation results. Some basic features predicted by the KdV equation are thus recovered, i.e., we find a growth of pulse amplitude as it propagates in the direction opposite to the gravity direction. Fine details like the amplitude-width soliton relation are, however, not recovered. The soliton amplitudewidth relation is qualitatively satisfied only for the case where we set gravitational acceleration $G=0$. For this particular case, the soliton deformation is small, and it is easier to make a soliton fit to the simulation curve. When we have a significant amount of reflected particles and at the same time formation of a trailing phase space vortex, it becomes difficult to find a proper identification of the width of a pulse and a local soliton property can no longer be demonstrated.

\section{CONCLUSION}

In the present study we analyzed weakly nonlinear ion acoustic sound pulses propagating in a gravitational plasma with an isothermal equilibrium. For this inhomogeneous system we can solve the linearized wave propagation problem in a fluid model analytically and find an amplitude increase of waves and pulses propagating in the antigravity direction. This is not a true instability [59] and has its origin in conservation of the flux of wave energy density in a medium with varying density. The potential of the wave has an increasing amplitude at increasing altitudes and becomes effective in reflecting particles. Ultimately, all wave energy is transformed to kinetic particle energy. The gravitational field thus serves as a "catalyst" in the transformation. We believe this to be 
a new observation. The system is energy conserving and we cannot gain particle energy exceeding what was present in the electrostatic pulse at $z=0$. Significant particle acceleration is found only in cases where we have large net energy in the injected pulses. If the ideas outline in the present study are applied to the polar ionosphere with vertical or nearly vertical magnetic field lines, we anticipate that relevant conditions are found for unstable E- or F-region conditions due to a two stream instability, for instance [60]. Significant perturbations can also be excited externally by, for instance, ionospheric heating experiments.

To give the problem an analytical basis we derived an approximate model in terms of a modified Korteweg-de Vries equation. We studied the propagation and deformation of soliton solutions for this equation. Some basic features of the numerical results are explained by the model equation also concerning the energy exchange between solitons and plasma ions. For the entire energy budget we have to include both the soliton and the nonsoliton parts, such as plateau and tail. For interaction with particles, we need to be concerned only with the soliton part since it has the dominant amplitude.

The numerical results show that some basic features of the $\mathrm{KdV}$ equation are supported, but illustrates also its shortcomings. As a test we first considered a limit where effects of gravity were ignored and found propagation of a moderate amplitude soliton shaped structure with a small damping. In this limit, the predictions from the $\mathrm{KdV}$ equation were in acceptable agreement with observations. We then increased the gravitational acceleration term and found the damping to be counterbalanced at $G=0.25$ resulting in a slow growth, and then for $G=0.5$ we find an initially exponential growth that saturates for large times in qualitative agreement with the analytical predictions but the detailed agreement was missing, in particular concerning the amplitude-width relation found for KdV solitons. It should be noted, however, that for practical reasons the parameters of the numerical simulations are rather extreme compared to those assumed in the analytical studies.

It is an essential element in the analysis that the linear energy propagation speed (here the ion sound speed) is constant for all vertical positions, independent of density. For a number of other wave types, also this speed is varying and the energy density flux then becomes a competition between several parameters. Phenomena and results similar to those studied here can be found for other inhomogeneous plasma conditions realizable in laboratory plasmas $[6,8-10,47]$, presheaths, for instance [30]. We note though that plasma sheaths near solid surfaces require models without assumptions of quasineutrality [30]. Such problems require a separate analysis. Conditions where a vertical current flow is forced from $z=0$ in the direction opposed to gravity are singular [61], and require also a separate analysis.

\section{ACKNOWLEDGMENTS}

P.G. was supported by the UK Science and Technology Facilities Council (STFC) Consolidated Grant (UCL/MSSL Solar and Planetary Physics, Grant No. ST/N000722/1), the UK STFC Consolidated Grant No. ST/M001334/1 (UCL Astrophysics), and the UK STFC Consolidated Grant No. ST/S000240/1 (UCL/MSSL-Physics and Astronomy Solar System).

\section{APPENDIX A: BOUSSINESQ TYPE EQUATIONS}

The $\mathrm{KdV}$ equation is explicitly derived for waves or pulses propagating in one direction, as evidenced by the operator $\partial / \partial t+C_{s} \partial / \partial z$ in the lowest order approximation. It is possible to obtain an equation which can account for bi-directional propagation, here given in dimensionless form [21],

$$
\frac{\partial^{2}}{\partial t^{2}} u-\frac{\partial^{2}}{\partial z^{2}} u-\frac{\partial^{4}}{\partial z^{4}} u-\frac{\partial^{2}}{\partial z^{2}} u^{2}=0 .
$$

The two first terms correspond to the classical sound equation as might be expected. The third term represents a dispersion, where we note that a term like $\partial^{4} u / \partial t^{2} \partial z^{2}$ might as well have been argued. The last term represents the nonlinearity. The equation does not have any significant advantage over the $\mathrm{KdV}$ equation, however, at least not as long soliton dynamics is an issue. The point is that two counterpropagating pulse overlap for only a small time, and do not manage to interact significantly. In case of overtaking interactions, the interaction time is much longer, and the interaction becomes significant. This limit is, however, well described by the KdV equation.

We can formulate a nonlinear equation that includes the Boussinesq equation for homogeneous conditions and at the same time accounts for the linear dispersion relation (4) obtained for the gravitational inhomogeneous system. This modified equation has the form,

$$
\frac{\partial^{2}}{\partial t^{2}} u-\frac{\partial^{2}}{\partial z^{2}} u-\frac{\partial^{4}}{\partial z^{4}} u-\frac{\partial^{2}}{\partial z^{2}} u^{2}=-g \frac{\partial}{\partial z} u,
$$

where $g$ is here a dimensionless measure of the gravitational acceleration. Equation (A2) can be reduced to our modified $\mathrm{KdV}$ equation, but on the expense of higher spatial and temporal derivatives, it has a wider range of applicability.

\section{APPENDIX B: EXPLICIT EXPRESSIONS FOR THE SERIES EXPANSION OF $G\left(U_{s}, \Psi(t)\right)$}

The lengthy series expansion [29] of $G\left(U_{s}, \Psi(t)\right)$ used in Sec. VII A is given here

or

$$
G\left(U_{s}, \Psi(t)\right)=2\left(\frac{e \Psi(t)}{M}\right)^{2} f_{0}^{(1)}\left(C_{s}\right)\left(1+4 \sum_{n=3}^{\infty} \frac{(2 n-1)(2 n-2)}{(2 n) !} \frac{f_{0}^{(2 n-3)}\left(U_{s}\right)}{f_{0}^{(1)}\left(U_{s}\right)}\left(\frac{2 e \Psi(t)}{M}\right)^{n-2}\right),
$$

$$
G\left(U_{s}, \Psi(t)\right)=2\left(\frac{e \Psi(t)}{T_{e}}\right)^{2} C_{s}^{4} f_{0}^{(1)}\left(C_{s}\right)\left(1+4 \sum_{n=3}^{\infty} \frac{(2 n-1)(2 n-2)}{(2 n) !} \frac{f_{0}^{(2 n-3)}\left(U_{s}\right)}{f_{0}^{(1)}\left(U_{s}\right)}\left(\frac{2 e \Psi(t)}{T_{e}}\right)^{n-2} C_{s}^{2 n-2}\right)
$$


where $f_{0}^{(m)}$ denotes the $m$ th derivative of $f_{0}(u)$. These series are subsequently used to rewrite relation (34). The lowest order approximation leads to an expression that can be integrated analytically.
[1] A. Pannekoek, Ionisation in stellar atmospheres, Bull. Astron. Inst. Netherlands 1, 107 (1922).

[2] S. Rosseland, Electrical state of a star, Mon. Not. Royal Astron. Soc. 84, 720 (1924).

[3] S. B. Ganguli, The polar wind, Rev. Geophys. 34, 311 (1996).

[4] A. W. Yau, T. Abe, and W. K. Peterson, The polar wind: Recent observations, J. Atmosph. Solar-Terrestrial Phys. 69, 1936 (2007).

[5] L. Stenflo, N. L. Tsintsadze, and T. D. Buadze, Solitary acoustic waves in weakly ionized gases, Phys. Lett. A 135, 37 (1989).

[6] H. L. Pécseli, Low Frequency Waves and Turbulence in Magnetized Laboratory Plasmas and in the Ionosphere (IOP Publishing, Oxford, 2016).

[7] K. C. Yeh and C. H. Liu, Theory of Ionospheric Waves, International Geophysics Series, Vol. 17 (Academic Press, New York/London, 1972).

[8] H. J. Doucet, W. D. Jones, and I. Alexeff, Linear ion acoustic waves in a density gradient, Phys. Fluids 17, 1738 (1974).

[9] N. D’Angelo, P. Michelsen, and H. L. Pécseli, DampingGrowth Transition for Ion-Acoustic-Waves in a Density Gradient, Phys. Rev. Lett. 34, 1214 (1975).

[10] N. D’Angelo, P. Michelsen, and H. L. Pécseli, Ion-acousticwaves in a density gradient, Z. Naturforsch. A 31, 578 (1976).

[11] H. L. Pécseli, Waves and Oscillations in Plasmas, 2nd ed. (Taylor \& Francis, London, 2020).

[12] P. Guio, S. Børve, H. L. Pécseli, and J. Trulsen, Low frequency waves in plasmas with spatially varying electron temperature, Ann. Geophys. 18, 1613 (2001).

[13] P. Guio and H. L. Pécseli, Weakly nonlinear ion waves in striated electron temperatures, Phys. Rev. E 93, 043204 (2016).

[14] C. O. Hines, Internal atmospheric gravity waves at ionospheric heights, Canadian J. Phys. 38, 1441 (1960).

[15] K. B. Dysthe and L. Stenflo, Atmospheric solitary waves, Phys. Scr. 12, 325 (1975).

[16] V. I. Karpman, Soliton evolution in the presence of perturbation, Phys. Scr. 20, 462 (1979).

[17] A. H. Nayfeh, Perturbation Methods (John Wiley \& Sons, New York, 1973).

[18] E. Fermi, On the origin of cosmic radiation, Phys. Rev. 75, 1169 (1949).

[19] G. B. Whitham, Linear and Nonlinear Waves (John Wiley \& Sons, New York, 1974).

[20] M. Kono and M. M. Škorić, Nonlinear Physics of Plasmas, Springer Series on Atomic, Optical, and Plasma Physics No. 62 (Springer, Heidelberg, 2010).

[21] P. G. Drazin and R. S. Johnson, Solitons: An Introduction (Cambridge University Press, Cambridge, 1989).

[22] S. Watanabe, Soliton and generation of tail in nonlinear dispersive media with weak dissipation, J. Phys. Soc. Jpn. 45, 276 (1978).

[23] S. Watanabe and N. Yajima, K-dV soliton in inhomogeneous system, J. Phys. Soc. Jpn. 53, 3325 (1984).

[24] V. Karpman and E. Maslov, Perturbation-theory for solitons, Zh. Eksp. Teor. Fiz. 73, 537 (1977) [Sov. Phys. JETP 46, 281 (1977)].
[25] V. I. Karpman, J. P. Lynov, P. Michelsen, H. L. Pécseli, J. J. Rasmussen, and V. A. Turikov, Modifications of plasma solitons by resonant particles, Phys. Fluids 23, 1782 (1980).

[26] M. Wadati and Y. Akutsu, Stochastic Korteweg-de Vries equation with and without damping, J. Phys. Soc. Jpn. 53, 3342 (1984).

[27] D. Parkinson and K. Schindler, Landau damping of long wavelength ion acoustic waves in a collision-free plasma with a gravity field, J. Plasma Phys. 3, 13 (1969).

[28] C. H. Liu, Long wavelength ion-acoustic waves in a magnetoplasma in a gravitational field, J. Plasma Phys. 4, 617 (1970).

[29] J. P. Lynov, P. Michelsen, H. L. Pécseli, and J. J. Rasmussen, Damping of Solitons by Reflected Particles, Tech. Rep. Ris $\emptyset-\mathrm{M}-$ 2168 (Risø National Laboratory, Roskilde, 1979).

[30] F. F. Chen, Introduction to Plasma Physics and Controlled Fusion, 3rd ed. (Springer, Heidelberg, 2016).

[31] E. Ott and R. N. Sudan, Nonlinear theory of ion acoustic waves with Landau damping, Phys. Fluids 12, 2388 (1969).

[32] J. W. VanDam and T. Taniuti, Nonlinear ion acoustic waves with Landau damping, J. Phys. Soc. Jpn. 35, 897 (1973).

[33] Y. Saitou and Y. Nakamura, Ion-acoustic shock waves undergoing Landau damping, Phys. Plasmas 10, 4265 (2003).

[34] A. Sikdar and M. Khan, Effects of Landau damping on finite amplitude low-frequency nonlinear waves in a dusty plasma, J. Theoret. Appl. Phys. 11, 137 (2017).

[35] Y. H. Ichikawa and T. Taniuti, Nonlinear wave modulation with account of the nonlinear Landau damping, J. Phys. Soc. Jpn. 34, 513 (1973).

[36] K. B. Dysthe and H. L. Pécseli, Non-linear Langmuir wave modulation in collisionless plasmas, Plasma Phys. 19, 931 (1977).

[37] J. P. Lynov, P. Michelsen, H. L. Pécseli, J. J. Rasmussen, K. Saeki, and V. Turikov, Observations of solitary structures in a magnetized, plasma loaded waveguide, Phys. Scr. 20, 328 (1979).

[38] T. Honzawa, Interaction of two ion acoustic solitons via reflected ions and amplification of trailing waves, Plasma Phys. Controlled Fusion 26, 449 (1984).

[39] K. B. Dysthe, H. L. Pécseli, and J. Trulsen, A statistical model for soliton particle interaction in plasmas, Phys. Scr. 33, 523 (1986).

[40] P. Guio, S. Børve, L. K. S. Daldorff, J. P. Lynov, P. Michelsen, H. L. Pécseli, J. J. Rasmussen, K. Saeki, and J. Trulsen, Phase space vortices in collisionless plasmas, Nonlin. Proc. Geophys. 10, 75 (2003).

[41] M. J. Poulos and G. J. Morales, Transport properties of a hollow pressure filament in a magnetized plasma, Phys. Plasmas 23, 092302 (2016).

[42] G. M. Molen, M. Kristiansen, and M. O. Hagler, CO2 laser beam refraction in a linear discharge plasma, App. Phys. Lett. 23, 601 (1973).

[43] K. B. Dysthe, E. Mjølhus, H. L. Pécseli, and L. Stenflo, Thermally stimulated scattering in plasmas, J. Appl. Phys. 57, 2477 (1985). 
[44] K. B. Dysthe, E. Mjølhus, H. L. Pécseli, and K. Rypdal, A thermal oscillating two-stream instability, Phys. Fluids 26, 146 (1983).

[45] P. N. Guzdar, P. K. Chaturvedi, K. Papadopoulos, M. J. Keskinen, and S. L. Ossakow, The self-focusing instability in the presence of density irregularities in the ionosphere, J. Geophys. Res. 101, 2453 (1996).

[46] F. Mottez, G. Chanteur, and A. Roux, Filamentation of plasma in the auroral region by an ion-ion instability: A process for the formation of bidimensional potential structures, J. Geophys. Res. 97, 10801 (1992).

[47] O. E. Garcia and H. L. Pécseli, Models for electrostatic drift waves with density variations along magnetic field lines, Geophys. Res. Lett. 40, 5565 (2013).

[48] W. M. Manheimer, Nonlinear development of an electron plasma wave in a cylindrical waveguide, Phys. Fluids 12, 2426 (1969).

[49] D. T. Blackstock, in Nonlinear Acoustics (Theoretical), 3rd ed. (McGraw-Hill, New York, 1972), Chap. 3, pp. 3-183.

[50] C. T. Chang, Shock wave phenomena in coaxial plasma guns, Phys. Fluids 4, 1085 (1961).

[51] P. Hart, Modified snowplow model for coaxial plasma accelerators, J. Appl. Phys. 35, 3425 (1964).

[52] K. Saeki, P. Michelsen, H. L. Pécseli, and J. J. Rasmussen, Formation and Coalescence of Electron Solitary Holes, Phys. Rev. Lett. 42, 501 (1979).
[53] H. L. Pécseli, J. Trulsen, and R. Armstrong, Formation of ion phase-space vortexes, Phys. Scr. 29, 241 (1984).

[54] I. B. Bernstein, J. M. Greene, and M. D. Kruskal, Exact nonlinear plasma oscillations, Phys. Rev. 108, 546 (1957).

[55] L. K. S. Daldorff, P. Guio, S. Børve, H. L. Pécseli, and J. Trulsen, Ion phase space vortices in 3 spatial dimensions, Europhys. Lett. 54, 161 (2001).

[56] L. Mandrake, P. L. Pritchett, and F. V. Coroniti, Electron beam generated solitary structures in a nonuniform plasma system, Geophys. Res. Lett. 27, 2869 (2000).

[57] C. Briand, A. Mangeney, and F. Califano, Coherent electric structures: Vlasov-Ampère simulations and observational consequences, J. Geophys. Res. 113, A07219 (2008).

[58] I. Y. Vasko, I. V. Kuzichev, O. V. Agapitov, F. S. Mozer, A. V. Artemyev, and I. Roth, Evolution of electron phase space holes in inhomogeneous plasmas, Phys. Plasmas 24, 062311 (2017).

[59] K. B. Dysthe, K. D. Misra, and J. K. Trulsen, On the linear cross-field instability problem, J. Plasma Phys. 13, 249 (1975).

[60] M. C. Kelley, The Earth's Ionosphere, Plasma Physics and Electrodynamics, International Geophysics Series Vol. 43 (Academic Press, San Diego, 1989).

[61] O. E. Garcia, E. Leer, H. L. Pécseli, and J. K. Trulsen, Magnetic field-aligned plasma currents in gravitational fields, Ann. Geophys. 33, 257 (2015). 